\section{Smooth Invariants of Four-Dimensional Manifolds and Quantum Field Theory}

\section{Gregory W. Moore}

In the past fifty years there has been a very vibrant interaction between physicists who study quantum field theories and string theories on the one hand and mathematicians investigating a wide variety of topics on the other hand. The interactions with mathematicians who study various aspects of (algebraic) geometry and (low-dimensional) topology have been particularly strong. Important mathematical discoveries such as (homological) mirror symmetry and the topological field theory interpretations of knot polynomials and knot homologies have their roots in physics. This talk will focus on one paradigmatic example of such an interaction between physicists and mathematicians: the relation between mathematical invariants of four-dimensional smooth manifolds and the physics of supersymmetric quantum field theories.

From the mathematical viewpoint, the differential topology of four-dimensional manifolds presents many interesting unanswered questions. There is nothing even close to a useful classification of simply connected, compact, oriented four-manifolds. Two important examples of smooth invariants of four-manifolds are based on the study of the moduli space of solutions to partial differential equations for connections on principal bundles over a four-manifold $X$. The Donaldson invariants, discovered in the 1980s, are based on the study of the renowned instanton equations. These are equations for a connection on a principal bundle over $X$ that state that the curvature is an anti-self-dual 2 -form. There is typically a moduli space of solutions to these equations, and Simon Donaldson showed that the intersection theory on this moduli space can be used to define differential topological invariants of X. The Seiberg-Witten invariants are equations associated to a spin-c structure on $X$. They are equations for a connection on a principal $U(1)$ bundle over $X$ (together with one other field). The moduli space of solutions to the Seiberg-Witten equations can also be used to define smooth invariants of $X$, known as Seiberg-Witten invariants. Since the moduli space of solutions to the Seiberg-Witten equations is much simpler than instanton moduli space (it is smooth and compact), it is easier to work with the Seiberg-Witten invariants. Happily, the Seiberg-Witten invariants turn out to be just as powerful, if not more powerful, than the Donaldson invariants.

In 1988, following some important questions and suggestions by Michael Atiyah, Edward Witten gave an interpretation of the Donaldson invariants as correlation functions of certain special operators in a certain quantum field theory known as "topologically twisted $\mathrm{N}=2$ supersymmetric Yang-Mills theory." However, the path integral formulation of the invariants could not be used as a practical means of evaluating the Donaldson invariants until aspects of the low-energy dynamics of that quantum field theory were understood better. This deeper understanding was achieved in 1994 when Nathan Seiberg, together with Witten, understood the nature of the groundstates of the theory in detail. After that breakthrough there was rapid progress, culminating in the formulation of the SeibergWitten invariants.

In fact, the Donaldson invariants can be expressed in terms of the Seiberg-Witten invariants. The relation was conjectured by Witten, at least for the case when $X$ has $b_{2}^{+}>1$. The relation was derived using physical methods (path integrals and effective field theory) by G. Moore and Witten in 1997. A crucial step in that derivation involves the so-called $u$-plane integral. This is a very subtle, finite-dimensional integral over the complex plane, closely related to the theta-lifts used in the theory of automorphic forms. The main part of the talk will focus on some progress achieved in the past few years continuing the approach to four-manifold invariants using quantum field theory. Time permitting, at least three results will be explained.

First, the topological twisting procedure of Witten can be extended to arbitrary quantum field theories with $\mathrm{N}=2$ supersymmetry. Around 2008 many new supersymmetric $\mathrm{N}=2$ field theories were discovered. Many of the new theories have the intriguing property that there is no known Lagrangian description (and probably no Lagrangian description exists). It has been a long-standing and natural question to ask if these new theories lead to new four-manifold invariants. Work with I. Nidaiev shows that, at least in the simplest of these non-Lagrangian theories, the answer is negative. Nevertheless, in the process of answering the question we still learn nontrivial facts about the Seiberg-Witten invariants. (An example is the superconformal simple type property of the Seiberg-Witten invariants.)

Gregory W. Moore is a professor in the Department of Physics and Astronomy at Rutgers University. His email address is gmoore@physics . rutgers. edu. For permission to reprint this article, please contact: reprint-permission aams.org.

DOI: https://dx.doi.org/10.1090/noti2000 
Second, it was already noted long ago by Moore and Witten and by Malmendier and Ono that for the case where $X$ is $C P^{2}$ and $S^{2} \times S^{2}$ the $u$-plane integral can be evaluated exactly using the theory of mock modular forms. Recent work with G. Korpas, J. Manschot, and I. Nidaiev has gone further, demonstrating that in fact for all four-manifolds with $b_{2}^{+}>1$ the $u$-plane integral can be expressed succinctly as the constant term in a Fourier expansion of a mock modular form (or of a mock Jacobi form).

Third, it was already noted by Donaldson in the $1980 \mathrm{~s}$ that the theory can be extended to families of four-dimensional manifolds. The Donaldson invariants are then no longer functions on the homology of the four-manifold $X$ but rather are functions on the homology valued in the cohomology of the classifying space BDiff $(X)$, where $\operatorname{Diff}(X)$ is the group of orientation-preserving diffeomorphisms of $X$. The talk will present some results from ongoing work with J. Cushing showing that such invariants can also be given an interpretation in quantum field theory, thus generalizing the formulation of the Donaldson invariants provided by Witten.

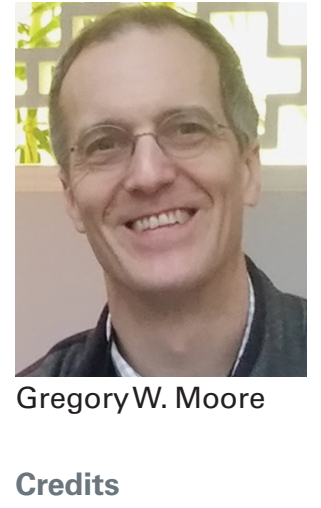

Author photo is courtesy of the author. 